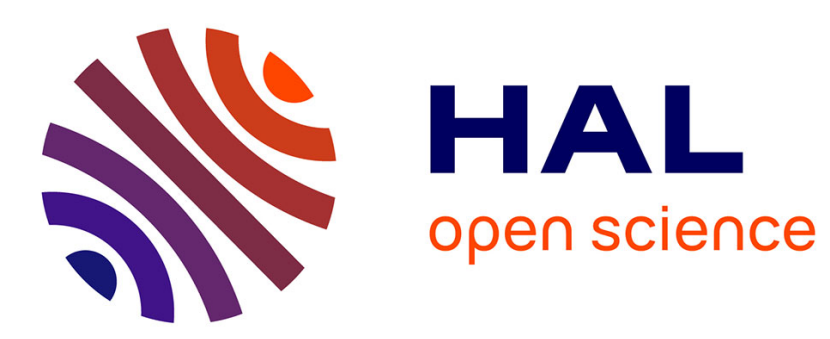

\title{
Analog computation of heterodyning with a voltage-biased Josephson point-contact \\ S.N. Erné
}

\section{To cite this version:}

S.N. Erné. Analog computation of heterodyning with a voltage-biased Josephson point-contact. Revue de Physique Appliquée, 1974, 9 (1), pp.243-247. 10.1051/rphysap:0197400901024300 . jpa-00243747

\section{HAL Id: jpa-00243747 https://hal.science/jpa-00243747}

Submitted on 1 Jan 1974

HAL is a multi-disciplinary open access archive for the deposit and dissemination of scientific research documents, whether they are published or not. The documents may come from teaching and research institutions in France or abroad, or from public or private research centers.
L'archive ouverte pluridisciplinaire HAL, est destinée au dépôt et à la diffusion de documents scientifiques de niveau recherche, publiés ou non, émanant des établissements d'enseignement et de recherche français ou étrangers, des laboratoires publics ou privés. 


\title{
ANALOG COMPUTATION OF HETERODYNING WITH A VOLTAGE-BIASED JOSEPHSON POINT-CONTACT
}

\author{
S. N. ERNÉ
}

Physikalisch-Technische Bundesanstalt, Braunschweig und Berlin, Germany

\begin{abstract}
Résumé. - Un système composé de 2 circuits résonnants reliés inductivement par un SQUID résistif a été étudié au moyen d'un calculateur analogique. Plusieurs résultats, qui présentent un intérêt particulier pour les applications dans le domaine de la réception des micro-ondes sont exposés. Ils se rapportent au spectre du signal de mélange, au déplacement de fréquence et au fonctionnement sans signal d'entrée.
\end{abstract}

Abstract. - A system consisting of two resonant circuits coupled inductively with a resistive SQUID was investigated using an analog computer. Here it is reported on several results of special interest for microwave receiver application, namely spectra of heterodyning products, frequency shifting and operation at zero input.

1. Introduction. - In the last years the interest in using Josephson junctions as mixers for microwave receivers has increased. Although the Josephson junction offers nearly ideal conditions for this application because of its highly non-linear currentvoltage-relationship and the very low operating temperature, progress has been rather slow due to the difficulties in understanding the interaction between the weak link and complex circuits.

The effect of microwave radiation on a voltagebiased weak link has been firstly investigated by Grimes and Shapiro [1]. If a voltage

$$
V_{\mathrm{j}}(t)=\bar{V}_{\mathrm{j}}+V_{\mathrm{in}} \cos \left(\omega_{\mathrm{in}} t+\theta_{\mathrm{in}}\right)
$$

is impressed to the junction one gets for an ideal weak link, with current-phase-relationship $I=I_{\mathrm{c}} \sin \varphi$, the following equation

$$
\begin{aligned}
I=I_{\mathrm{c}} \sum_{m=-\infty}^{\infty} J_{m}(2 & \left.\pi \frac{V_{\text {in }}}{\omega_{\text {in }}} \frac{2 \mathrm{e}}{h}\right) \times \\
& \times \sin \left[\left(\omega_{\mathrm{j}}+m \omega_{\text {in }}\right) t+\theta_{0}+m \theta_{\text {in }}\right]
\end{aligned}
$$

where $\omega_{\mathbf{j}}=\frac{2 \mathrm{e}}{\hbar} \bar{V}_{\mathbf{j}}$ denotes the Josephson frequency and $V_{\mathbf{j}}$ the voltage at the junction. Since the junction current contains components with the difference frequencies $\omega_{\mathrm{IF}}=\omega_{\mathrm{j}}-|m| \omega_{\mathrm{in}}$ this equation indicates the applicability of a weak link as mixer and local oscillator in a heterodyne receiver.

Experimental investigations, [2], [3], have shown a more complex behaviour in real arrangements and their results cannot be explained by this simple model.

A first approach to expand the model has been made by Hamilton [4] taking into account the impe- dance of the dc-voltage source and of the radio-frequency source.

2. The model of the mixer. - We here study a circuit system shown in figure 1 containing essential features of a heterodyne receiver using a resistive SQUID coupled with two resonant circuits. One

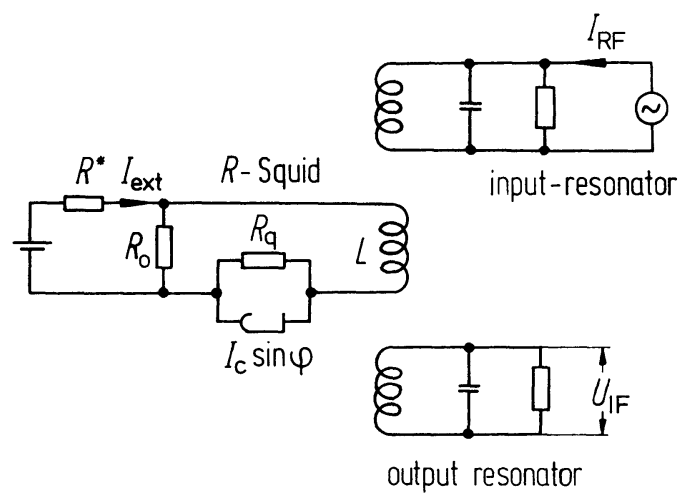

Frg. 1. - Model of the resistive SQUID mixer.

circuit is needed to couple the input signal, the other to decouple the Intermediate Frequency signal. The junction is working as a local oscillator as well as a mixer. To tune the oscillator the junction is voltage biased by shunting the weak link with a resistance $R_{0}\left(\approx 10^{-6} \Omega\right)$. All the inductances of the resistive SQUID circuit are combined and will be denoted by $L$ : it couples the resistive SQUID inductively with both of the resonators.

The weak link is characterized by the maximum Cooper pair current $I_{\mathrm{c}}$ and the quasiparticle resistance $R_{\mathrm{q}}$; furthermore we assume a point-contact 
with negligible capacity and a sinusoidal pair currentphase relationship.

3. The system equations. - To develop the system equations we can modify the equivalent circuit as shown in figure 2.

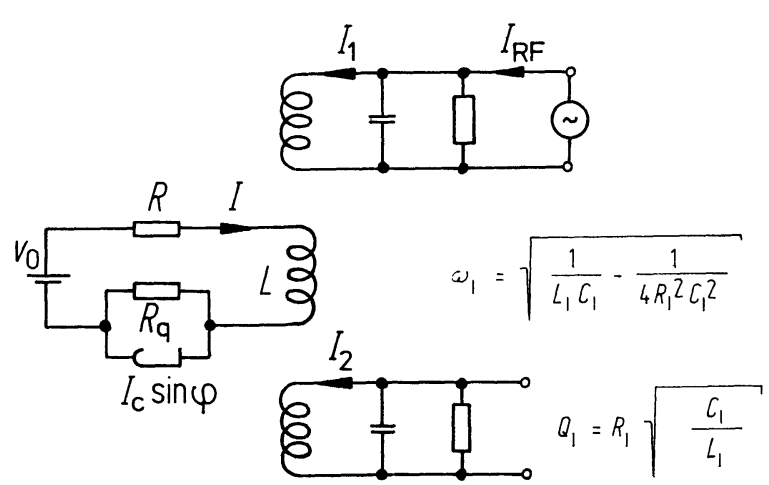

FIG. 2. - Modified equivalent circuit of the resistive SQUID, index 1 denotes the input circuit, index 2 the output circuit.

The current $I$ flowing through the contact is distributed on the superconducting and normal conducting paths according the following equation

$$
I(t)=I_{\mathrm{c}} \sin \frac{2 \mathrm{e}}{\hbar} \int_{0}^{t} V_{\mathrm{j}}\left(t^{\prime}\right) \mathrm{d} t^{\prime}+\frac{1}{R_{\mathrm{q}}} V_{\mathrm{j}}(t)
$$

where

$$
V_{\mathrm{j}}(t)=\frac{\hbar}{2 \mathrm{e}} \frac{\mathrm{d} \varphi}{\mathrm{d} t}
$$

denotes the voltage at the weak link and $\varphi$ is the phase difference of the Ginzburg-Landau parameters coupled at the junction.

Neglecting the direct coupling between input and output resonators, the corresponding system of differential equations can be set up using Kirchhoff's laws :

$$
\begin{aligned}
& \frac{\mathrm{d} I}{\mathrm{~d} t}-\frac{M_{1}}{L} \frac{\mathrm{d} I_{1}}{\mathrm{~d} t}-\frac{M_{2}}{L} \frac{\mathrm{d} I_{2}}{\mathrm{~d} t}+\frac{V_{0}}{L}+ \\
& \quad+\frac{R_{\mathrm{q}}}{L}\left(1+\frac{R}{R_{\mathrm{q}}}\right) I-\frac{R_{\mathrm{q}}^{\mathrm{I}}}{L} I_{\mathrm{c}} \sin \varphi=0 \\
& \frac{\mathrm{d}^{2} U_{1}}{\mathrm{~d} t^{2}}+\frac{1}{R_{1} C_{1}} \frac{\mathrm{d} U_{1}}{\mathrm{~d} t}+\frac{1}{L_{1} C_{1}} U_{1}= \\
& =\frac{M_{1}}{L_{1} C_{1}} \frac{\mathrm{d} T}{\mathrm{~d} t}+\frac{1}{C_{1}} \frac{\mathrm{d} I_{\mathrm{rf}}}{\mathrm{d} t} \\
& \frac{\mathrm{d}^{2} U_{2}}{\mathrm{~d} t^{2}}+\frac{1}{R_{2} C_{2}} \frac{\mathrm{d} U_{2}}{\mathrm{~d} t}+\frac{1}{L_{2} C_{2}} U_{2}=\frac{M_{2}}{L_{2} C_{2}} \frac{\mathrm{d} I}{\mathrm{~d} t}
\end{aligned}
$$

where

$$
M_{\mathrm{i}}=k \sqrt{L L_{\mathrm{i}}} \quad(i=1,2)
$$

$k=$ coupling constant.
The first equation determines the current flowing in the resistive SQUID. The last two equations are related to the resonators.

By reasons of computing technique the system of differential equations has been transformed. The final form of the equation system is as follows :

$$
\begin{aligned}
& I=\frac{M_{1}}{L} I_{1}+\frac{M_{2}}{L} I_{2}- \\
& \quad-\frac{R_{\mathrm{q}}}{L} \int_{0}^{t}\left[\frac{V_{0}}{R_{\mathrm{q}}}+\left(1+\frac{R}{R_{\mathrm{q}}}\right) I-I_{\mathrm{c}} \sin \varphi\right] \mathrm{d} t \\
& C_{1} \frac{\mathrm{d} U_{1}}{\mathrm{~d} t}+\frac{1}{R_{1}} U_{1}+\frac{1}{L_{1}} \int_{0}^{t} U_{1} \mathrm{~d} t=\frac{M_{1}}{L_{1}} I+I_{\mathrm{rf}} \\
& C_{2} \frac{\mathrm{d} U_{2}}{\mathrm{~d} t}+\frac{1}{R_{2}} U_{2}+\frac{1}{L_{2}} \int_{0}^{t} U_{2} \mathrm{~d} t=\frac{M_{2}}{L_{2}} I .
\end{aligned}
$$

Furthermore it has to be taken into account for the programming the relationship

$$
\frac{\mathrm{d} \varphi}{\mathrm{d} t}=\frac{2 \mathrm{e}}{\hbar} R_{\mathrm{q}}\left(I-I_{\mathrm{c}} \sin \varphi\right) .
$$

This differential equation was programmed on basis of a technique developed separately [5].

The patching diagram is shown in figure 3. A time normalization factor of $10^{9}$ was chosen as the optimal compromise between the desired speed and the available precision of computation. Thus a frequency of $10 \mathrm{~s}^{-1}$ for the analog model has been equivalent to a real frequency of $10 \times 10^{9} \mathrm{~s}^{-1}$.

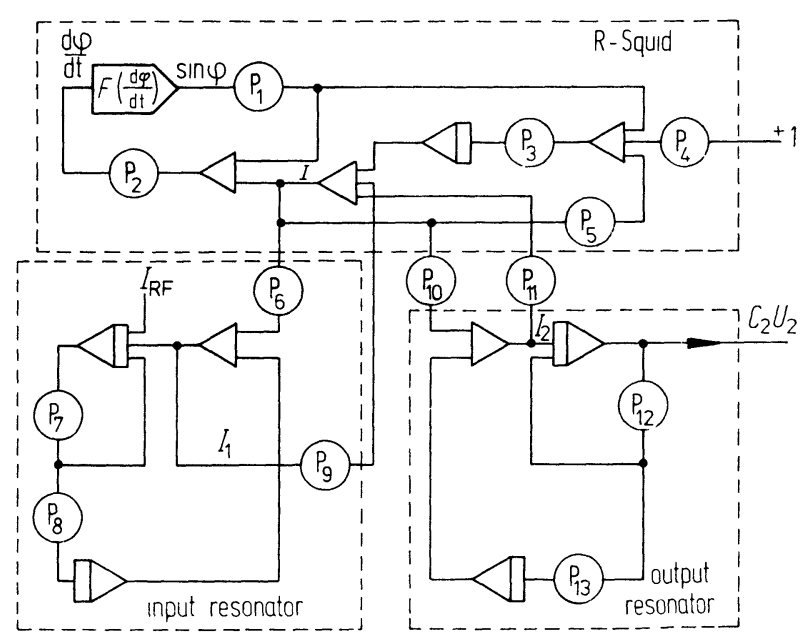

FIG. 3. - Patching diagram.

4. Results. - It has been our main intention to get results comparable with experimental ones. For this purpose a special set of parameters $\left(I_{\mathrm{c}}=0.3 \mathrm{~mA}, R_{\mathrm{q}}=0.2 \Omega, L=2 \times 10^{-10} \mathrm{H}\right.$, $Q_{1}=30, Q_{2}=5, R_{0}=10^{-6} \Omega$ ) had to be chosen. Furthermore as a general result it could be observed that the characteristic features are independent of the special set of parameters in a wide range. 
4.1 Spectrum. - To investigate the main behaviour of the system, spectra were plotted in compliance with the experimental results [3] : a signal with a constant frequency $f_{1}=\omega_{1} / 2 \pi$ was fed into the input circuit, then the frequency of the Josephson oscillator was varied between zero and values above $f_{1}$ by sweeping the junction voltage. The voltage at the output circuit was measured using broadband voltmeter and plotted as a function of $V_{0}$.

In figure 4 we see such a spectrum. The computed spectral structure is more complex than the one expected in a first approximation using the impressed voltage model. Because of the interaction between the inductivity and the weak link the current $I$ presents a high content of high order components : thereby the heterodyne frequencies are determined by following equation

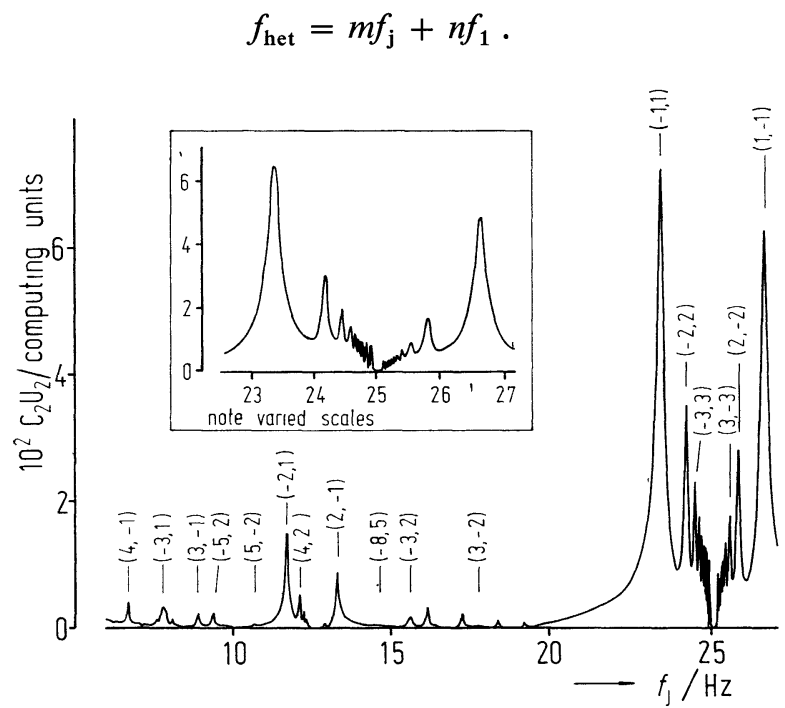

FIG. 4. - Output signal amplitude as function of $f_{\mathrm{j}} ; f_{1}=25 \mathrm{~Hz}$, $f_{2}=1.6 \mathrm{~Hz},\left(I_{\mathrm{re}}\right)_{\mathrm{pp}}=50 \mu \mathrm{A}$. The inset shows the part in proximity of $f_{1}$ with varied scales.

In the figure some of the lines are denoted by the corresponding integers $m$ and $n$ in the form $(m, n)$. The part of special interest in proximity of the frequency $f_{1}$ is expanded shown in the inset of figure 4 . In this region the lines of the spectrum, as far as they can be measured, appear at

$$
f_{1} \pm \frac{1}{q} f_{2}
$$

where $q=m=n$.

The appearance of such a fine structure was mentioned in a recent work of Finnegan and Wahlsten [6], whereas an agreement with the result of Fife and Gygax [3] is only partially given.

As another remarkable effect one observes the asymmetrical shape of the structure in the inset of figure 4 . This asymmetry with regard to $f_{1}$ depends on the amplitude of the input signal.

In order to understand this effect, the power exchange $P$ between input circuit and resistive SQUID has been computed. The signs were chosen in such a manner as to make $P$ positive if power is transferred from the resistive SQUID to the input circuit. $P$ as a function of $f_{\mathrm{j}}=V_{0} 2 \mathrm{e} / h$ for $I_{\mathrm{rf}}=0$ and $\left(I_{\mathrm{rf}}\right)_{\mathrm{pp}}=10 \mu \mathrm{A}$ is shown in figure 5 ; for a better understanding the difference $\Delta P$ between $P_{\mathrm{a}}$ and $P_{\mathrm{b}}$ is shown separately. The values of $\Delta P$ near $f_{1}$ could not be computed since the errors have been too large for reasonable integration times. For $f_{\mathrm{j}}<f_{1}$ additional power is transferred from the weak link to the input circuit, thereby the amplitude of the heterodyne signals are greater than the corresponding lines for $f_{\mathrm{j}}>f_{1}$.
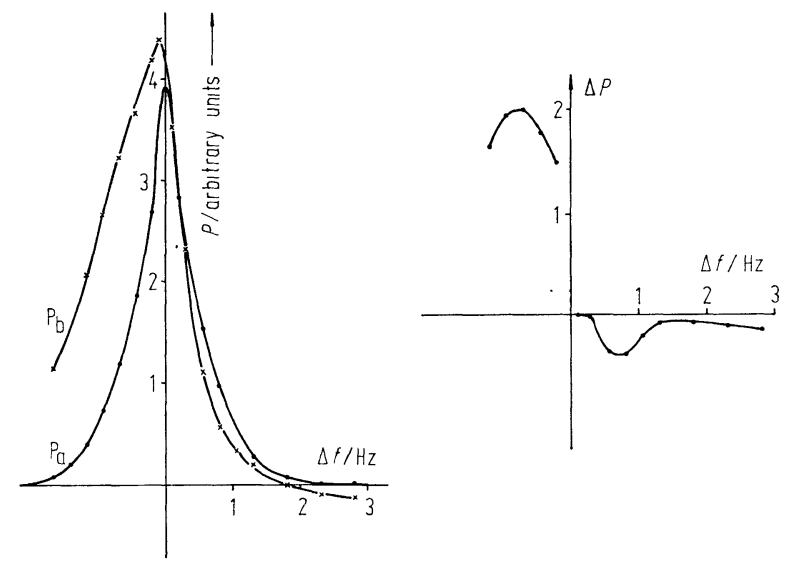

FIG. 5. - Power exchanged between input circuit and resistive SQUID. $f_{1}=25 \mathrm{~Hz} ; P_{\mathrm{a}}$ is plotted at $\left(I_{\mathrm{rr}}\right)_{\mathrm{pp}}=10 \mu \mathrm{A}, P_{\mathrm{b}}$ at $I_{\mathrm{rf}}=0$.

4.2 The FREQUenCY ShIFT. - The results discussed so far are valid for the small-signal range. The results of the analog computation show a dependence of the output signal frequency on the input signal amplitude for large input signals : by increasing the amplitude of the input signal the frequency of the output signal is decreased. This behaviour can be explained by the modification of the spectral distribution of the current of the resistive SQUID. Here only the shifting of the spectral components in the proximity of the resonance frequency $f_{2}$ is studied.

To describe this effect we introduce a correcting factor $\left[1-K\left(I_{\mathrm{rf}}\right)\right]$ into the equation defining the heterodyne frequency :

$$
\omega_{\mathrm{IF}}=\left[1-K\left(I_{\mathrm{rf}}\right)\right]\left( \pm \omega_{\mathrm{in}} \pm \frac{\left\langle V_{\mathrm{j}}>2 \mathrm{e}\right.}{\hbar}\right) .
$$

$K\left(I_{\mathrm{rf}}\right)=\Delta T / T_{0}$ is the deviation $\Delta T$ of the period of the IF signal related to $T_{0}$, which is the period for small signals. In figure $6 K\left(I_{\mathrm{rf}}\right)$ is shown for different values of $I_{\mathrm{c}}$ : we can recognize that for small signals the deviation is negligible and of the same magnitude of the computation error, whereas it amounts to a few percent for large input signals. These values are in agreement with the experimental results of Ulrich [7]. 


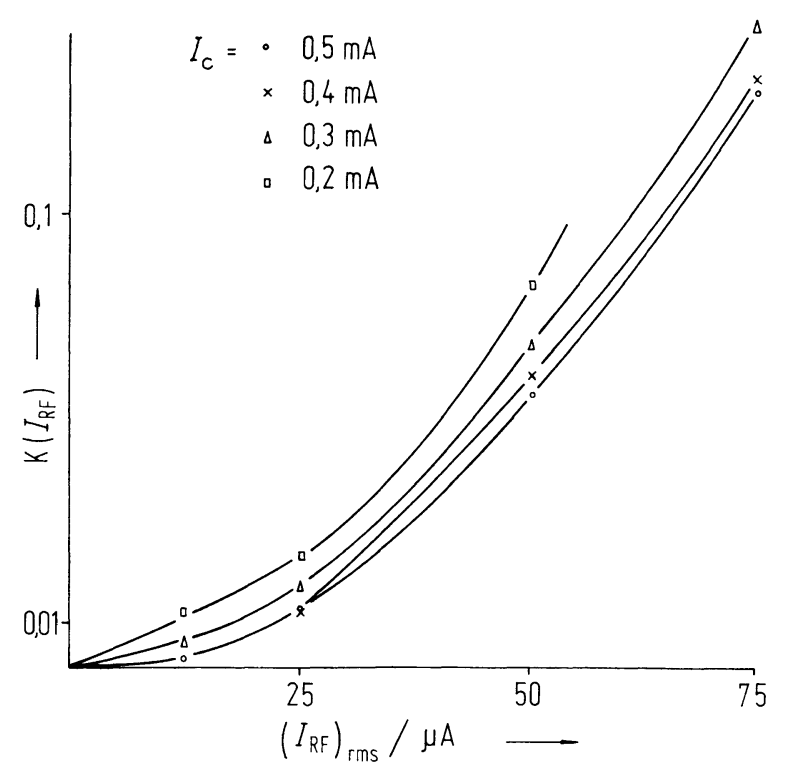

FIG. 6. - Frequency shift factor $K\left(I_{\mathrm{rf}}\right)$ for various values of the critical current $I_{\mathrm{c}}$.

4.3 $I_{\mathrm{rf}}=0 .-$ The effect of the interaction between input resonator and the resistive SQUID superimposed on the heterodyning is observable in the case of extreme low level input signals. In order to investigate this effect spectra were recorded without any input signal.

The computation has shown that, in the case of a coupling of the weak link with a resonator, there arises an output signal at bias voltages $V_{0} \approx f_{1} h / 2 \mathrm{e}$ even without any rf-input.

The interaction between the weak link and the resonator is describable as a "frequency pulling " effect of the same kind as has been found experimentally by Ulrich [7]. Here it is influenced by the output resonator. Whereas the Josephson oscillator frequency varies continuously between the Josephson

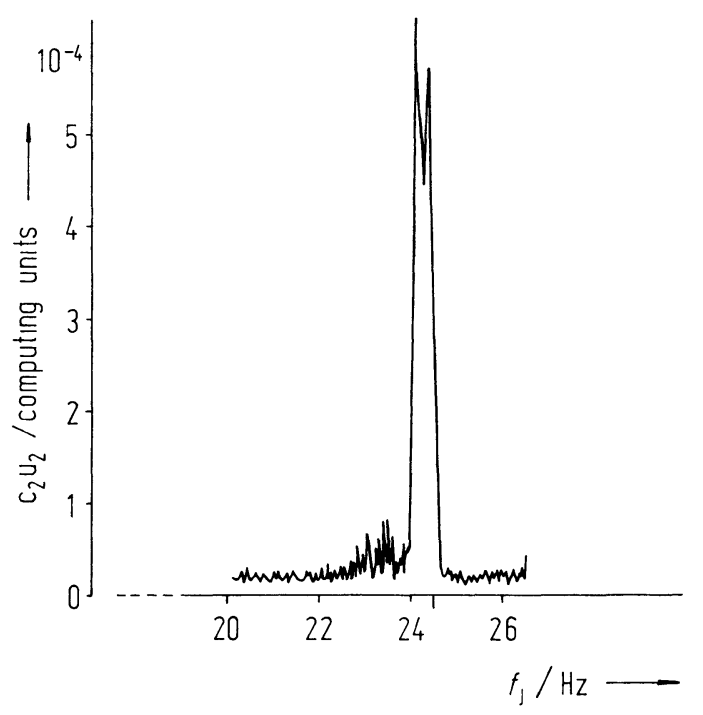

FIG. 7. - Output signal amplitude as function of $f_{\mathrm{j}}$ by $I_{\mathrm{rf}}=0$; $Q_{1}=30, Q_{2}=5$. self-oscillation frequency $f_{0}$ and the resonance frequency of the input circuit $f_{1}$, an amplitude modulated signal appears in the input resonator. The component with frequency $f_{2}$ appears at the output. The spectrum for this case is shown in figure 7 .

4.4 THE DEPENDENCE ON THE SYSTEM PARAMETERS. The behaviour of the system was investigated with regard to the parameters $I_{\mathrm{c}}, R_{\mathrm{q}}$ and the loop inductance of the resistive SQUID.

The computation has shown that the combined effect of $R_{\mathrm{q}}$ and $L$ results in the low pass filter effect, which can be derived using the first system equation. It is this effect which defines the upper frequency limit for the signal frequency.

The influence of $I_{\mathrm{c}}$ was studied in a special case. The amplitude of the first spectral line for $f_{\mathrm{j}}=f_{1}-f_{2}$ was measured as a function of $I_{\mathrm{c}}$ applying a small input signal of constant amplitude. The results are shown in figure 8.

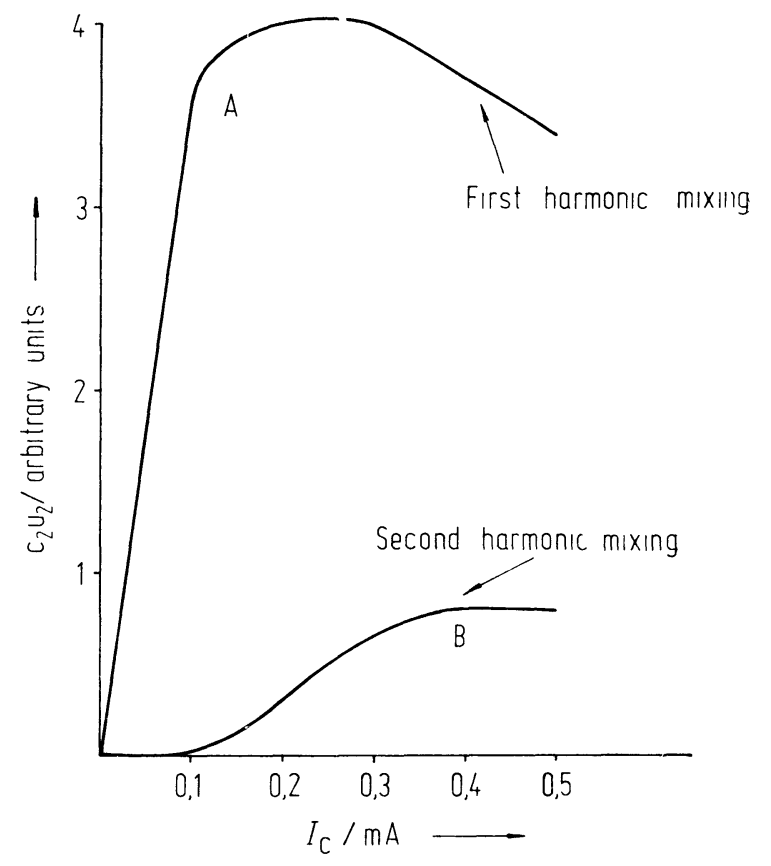

FIG. 8. - Amplitude of the output signal for constant input signal amplitude in dependence on critical current $I_{c}$.

A drop of the output signal occurs for large values of $I_{\mathrm{c}}$, in parallel the amplitudes of the high order components of the current $I$ are increased in the case $I_{\mathrm{c}} \gg \varphi_{0} / 2 \pi L$. In consequence, if the mixing is performed by the second order harmonic corresponding to the following equation

$$
\omega_{\text {het }}= \pm \omega_{1} \pm 2 \frac{V_{0} 2 \mathrm{e}}{\hbar}
$$

the output signal becomes appreciable only for $I_{\mathrm{c}} \gg \varphi_{0} / 2 \pi L$. This behaviour is shown in plot $\mathrm{B}$ of the figure. 
5. Conclusion. - Finally it can be concluded that the simple model studied here reflects on the essential features of heterodyne receivers using a Josephson junction as local oscillator and mixer.
Acknowledgments. - The author wishes to thank Dres. H.-D. Hahlbohm and H. Lübbig for stimulating discussions and Mr. H. Luther and Mr. P. Jäkel for assistance in computing problems.

\section{References}

[1] Grimes, C. C. and Shapiro, S., Phys. Rev. 169 (1968) 397. [5] Hahlbohm, H. D. and Luther, H., Elektron. Rechenanl.

[2] Zimmermann, J. E., J. Appl. Phys. 41 (1970) 1589. 13 (1971) 167.

[3] Fife, A. A. and Gygax, S., J. Appl. Phys. 43 (1972) 2391.

[6] Finnegan, T. F. and Wahlsten, S., Appl. Phys. Lett. 21 (1972) 541.

[4] Hamilton, C. A., J. Appl. Phys. 44 (1973) 2371

[7] Ulrich, B. T., Phys. Lett. 42A (1972) 119. 